\title{
La Gestión Compleja: De la Jerarquía a las Redes Complejas y la Heterarquía
}

\author{
Carlos Eduardo Maldonado \\ Profesor titular \\ Facultad de medicina \\ Universidad El Bosque \\ maldonadocarlos@unbosque.edu.co \\ ORCID: http://orcid.org/0000-0002-9262-8879
}

Recepción: 06/09/2021

Aceptación: 15/10/2022

\section{Resumen}

Este artículo estudia cómo es posible una gestión del mundo modo complejo. Una gestión semejante está vinculada a la naturaleza, y no a los sistemas humanos; ciertamente no en la manera como atávicamente han sido entendidos a lo largo de la historia de la humanidad occidental. Una gestión modo complejo consiste en el distanciamiento de sistemas jerárquicos, piramidales y centralizados hacia dinámicas de heterarquía y redes complejas. La tesis central de este texto es que una gestión modo complejo es gestión que no sabe de control, y por ello mismo se despliega en términos de autoorganización o, lo que es equivalente, de confianza. Los dos ejes que aparecen como conductores hacia, o fundamentos de, una gestión modo compleja, las redes complejas y la heterarquía implican que la administración en sentido amplio debe poder saber de antropología, epigrafía, entnología y etnografía, en fin, de historia y microhistoria, y no ya solamente de las aproximaciones tradicionales, todas de origen distintivamente ingenieril.

Palabras clave: Complejidad, no-linealidad, vida, democracia, confianza

\section{Abstract}

This paper studies how management is possible understood under the scope of complexity theory, i.e. complexity science. Such management goes hand in hand with a relation and an understanding of nature, not just of human affairs, which is the way in which management has been classical grasped along the history of the western world. Complexity management consists thus in distancing form hierarchic, pyramidal and centralized systems towards dynamics characterized by complex networks and heterarchy. The claim here is that complexity management does not know of control and, thereafter, it leads toward self-organization or also to trust. The two axis that appear ad ground or also al leading threads for a complexity management, namely complex networks and heterarchy, entail that management at large must know about anthropology, 
epigraphy, ethnology and ethnography, micro-history and not just about the typical approaches that emerge from engineering.

Key Words: Complexity, non-linearity, life, democracy, trust

\section{Introducción}

Las ciencias de la complejidad se encuentran aún lejos de convertirse en parte de la corriente principal de pensamiento (mainstream science). Ciertamente es evidente la forma como el lenguaje de la complejidad se viene permeando a través de diversas prácticas, ciencias y disciplinas, y cómo existe una masa crítica que crecientemente se interesa por las ciencias dela complejidad. Asimismo, el número de simposios, seminarios, congresos, conferencias y demás dedicados al tema es creciente alrededor del mundo, al tiempo que se multiplican las publicaciones en el área. Cada vez más aparecen editoriales con colecciones y revistas especializadas en el tema. Todo ello, motivo de orgullo para la comunidad de complejólogos. Pues bien, una razón fuerte por la cual la complejidad está aún lejos de formar parte de la vida cotidiana de la sociedad, las organizaciones, las instituciones, en el sector público, el privado o en el tercer sector se debe a la implementación misma de la complejidad, por así decirlo. No en vano, recurrentemente, en particular en los países de habla hispana, emerge la pregunta acerca de la forma como la complejidad se aplica o se gestiona o se incorpora, haciendo pensar siempre en lo comporta una instrumentalización de la complejidad. Se trata de preocupaciones legítimas, en particular cuando se las ve con los ojos de pasado o, lo que es lo mismo, con los ojos de un ánimo de concreción y aplicación de ciencias, teorías, ideas y comprensiones. Este texto busca allanar este camino.

El hilo conductor es el estudio de los temas y problemas relativos a la gestión, un tema que compete por igual a la política y la economía, a las finanzas y la administración, a la cosa pública y a las empresas, en fin, a las formas de organización humanas en cualquier momento y lugar, cualquier que sea su tamaña o su finalidad. Era exactamente esto lo que designaba a la política en el mundo antiguo: los asuntos que competen a la Polis, en fin, al convivio, mucho antes o bien mucho más allá de los temas relativos a los sistemas políticos, los regímenes políticos y otros próximos y semejantes. No en última instancia, el tema atañe igual y necesariamente a la organización del aprendizaje de los seres humanos, esto es, a la educación.

La tesis que defiende este texto afirma, negativamente, que las formas jerárquicas de organización no resuelven mayor cosa; solo las empeora, algo que con el tiempo se hace evidente (Mezza-García, Maldonado, 2015). Baste si no mirar a la propia historia de la civilización occidental, distintivamente organizada, a lo largo de más de 2500 años en torno a sistema verticales, jerárquicos, piramidales; en la religión y en la política, en los asuntos militares y en la educación, en fin, en las penurias y necesidades tanto como en los tiempos de paz. Sin embargo, la tesis formulada admite una comprensión positiva o afirmativa. Esta sostiene que es posible, e incluso necesario, una transformación radical de las formas jerárquicas de organización hacia formas que cuiden y exalten la vida. Pues bien, el modo como una transformación semejante es posible es a través de redes complejas o también de heterarquías. El resto de artículo está dedicado a mostrar lo que implican las redes complejas y la heterarquía, tanto como a establecer, exactamente, cómo es una gestión compleja o una gestión heterárquica.

Ciertamente, de manera reciente hay una bibliografía acerca de temas organizacionales y heterarquía (Aime et al., 2014; Chandrasekar-Smitha, 2017; Schoelhammer, 2020; Annushkina, Regazz, 2020; Pini-Fitzsimmons et al., 2021). Sostengo que no es precisamente en esta dirección que apunta este trabajo. Por el contrario, más originariamente, la heterarquía es un tema que nace en, y se alimenta de, la antropología (Hüsken, 2019; Cayón, 2020), pero con ella, entonces también derivativamente, en conexión estrecha con la etnografía, la etnohistoria, la epigrafía, la etnología, la arqueología y la historia (Crumley, 2005).

Ahora bien, la comunidad de complejólogos no ha trabajado hasta la fecha en profundidad el tema de la heterarquía. Existen razones precisas que explican esta circunstancia, que tienen que ver con el acentuado interés por las redes complejas, al margen justamente de las heterarquías; en otras palabras, por un fuerte acento computacional al margen de un trabajo también con la antropología en sentido amplio (Barrat et al., 2008). La novedad de este artículo estriba en la conexión entre redes complejas y heterarquía, por consiguiente, entre modelamiento y simulación y antropología, historia y política, en el marco de las ciencias de la complejidad.

Sin ambages, poner en un mismo plano a las redes complejas con la heterarquía y los temas de gestión es una contribución de corte latinoamericano (Ugalde y Landázuri Narváez, 2016), y más auténticamente, de origen precolombino (Kurnik and Baron, 2016). Fueron los pueblos, culturas, sociedades y civilizaciones precolombinas las que, en marcado contraste con Occidente, sabían y saben de heterarquía.

Los argumentos que sostienen a la tesis enunciada son tres. En primer lugar, se trata de señalar los peligros de los sistemas jerárquicos. Mientras que clásicamente, de manera abierta o tácita, se afirmó que los sistemas jerárquicos son evidentes y necesarios, este 
texto argumentará que son antinaturales, y por tanto, violentos. El segundo argumento se concentra en las redes complejas con una doble finalidad; de un lado, cribar de cualquier buen entendimiento el holismo, que es, hay que decirlo expresamente, mala ciencia. Y de otra parte, se trata de comprender por tanto cómo las redes complejas permiten un modo de gestión que sea exaltador y posibilitador de vida. Sobre esta base, el tercer argumento se concentra en el estudio de la heterarquía, y establece de manera explícita cómo ésta se integra con el estudio de los fenómenos y sistemas de complejidad creciente. Una mirada a la antropología se impone, y se destacan los beneficios de una mirada semejante. Al final, la conclusión señala que una gestión compleja es gestión de vida, no de materiales, dineros, recursos, y demás. Las redes complejas y las heterarquías son exactamente el modo como funciona y se estructura la naturaleza.

\section{Los peligros de los sistemas jerárquicos}

De manera tradicional, Occidente concibió la estructura misma del universo y la naturaleza como un sistema jerárquico y centralizado y, consiguientemente, organizó la sociedad y todos los asuntos humanos de la misma manera (Maldonado, 2020a). Esta historia no se reduce únicamente a ese momento puntual que es la hibridación entre Atenas, Roma y Jerusalén; se encuentra, en realidad, desde la misma concepción de Occidente, hacia el año 7.000-5.000 a.e.v. Específicamente, se trata de la historia que, desde los orígenes del neolítico, incluye al antiguo Egipto, a Persia, Mesopotamia, los Fenicios y los Hititas (Lévèque, 2012).

Pues bien, la historia de la estructuración de la sociedad en términos jerárquicos, piramidales, centralizados coincide, plano por plano, con la historia misma de la violencia, la exclusión, las inequidades y las desigualdades. En efecto, toda la historia de Occidente se condensa en la siguiente ecuación:

$$
H=1 / N
$$

en la que $\mathrm{H}$ expresa a la especie humana, y $\mathrm{N}$ a la naturaleza, de tal suerte que el ser humano se concibe como exterior y superior a la naturaleza, y reduce a esta a un medio que existe en función de los intereses, necesidades y deseos humanos. Una concepción semejante, mucho antes del Génesis en el Libro de los Libros, se encuentra en la Política de Aristóteles. Sólo que la "naturaleza" es el título genérico que expresa a todo lo ajeno, externo, en fin, lo otro de lo propio, y que consiguientemente designa a los úeb,os primitivos, a las culturas lejanas, a las civilizaciones extrañas. Dicho sin más, la "naturaleza" habrá de incluir a otros pueblos, culturas, sociedades, civilizaciones y al interior de la ciudad occidental, a los pobres, desplazados, necesitados, reseñados, extranjeros y excluidos. Que el mundo se hubiera estructurado jerárquicamente comportó una noción de ascenso y descenso, de pruebas y mecanismos de selección, de asimilación y cooptación, en fin, de distanciamiento y separación. Todo lo demás, fue historia, hasta el presente. Erróneamente, Occidente creyó ella misma, y le quiso hacer creer a otros pueblos y sociedades, que así eran las cosas y no podrían ser de otro modo. A los pueblos que vivieron de otra forma, Occidente terminó robándoles la historia, la memoria (Goody, 2006), en fin, su propio ser.

Para decirlo en otras palabras, una manera semejante de concebir el universo, la realidad, la naturaleza y el mundo, supuse la superposición de la cultura sobre -y en contraste con- la naturaleza. En una palabra: se trató de una concepción antinatural, dualista, binaria, bivalente.

Puede decirse que la idea de sistemas jerárquicos es concomitante con el descubrimiento y el despliegue del individualismo (Sheldrake, 2012). Sería interesante rastrear estos orígenes; aquí, por razones de espacio, digamos que se encuentran exactamente en las semillas mismas constitutivas de la humanidad occidental, si bien el individuo y el individualismo no ha sido descubierto una sola vez, sino varias a lo largo de la historia de la civilización occidental (Gauthier, 1998; Foccroulle et al., 2005; Macpherson, 2005). Una lectura reciente de este mismo proceso afirma que el descubrimiento del individualismo coincide por completo con la erección de la sociedad patriarcal.

Como quiera que sea, la imposición de sistemas jerárquicos es una sola y misma cosa con la imposición de un pensamiento analítico; esto es, (des)agregativo, composicional, clasificatorio, que cree en y trabaja con categorías. Las categorías son siempre clasificatorias y no en última instancia jerarquizantes. Este tipo de pensamiento termina por hacer creer que hay cosas que son más importantes que otras, cree en necesidades en contra de fenómenos contingentes, desplaza a lugares secundarios aquello que no es necesario y entonces subsidiario, en fin, ordena las cosas de modo piramidal y centralizado. Basta con echar una mirada a la historia para entender las consecuencias de una mentalidad semejante. Digámoslo en una palabra: una estructuración semejante no sabe nada de complejidad, esto es, de redes complejas, de sistemas autoorganizativos, en fin, de no-linealidad. Todo responde a un espíritu distintivamente lineal, secuencial, jerárquico (Mezza-García, Maldonado, 2015).

Occidente no supo en toda su historia de otra forma que de organización vertical de las cosas. Así explicó, especialmente, en el medioevo, la estructura del cielo, compuesto por Dios, los arcángeles, los ángeles, los santos, los querubines, y luego las almas mortales, 
en orden descendiente. Asimismo, concibieron Platón y Aristóteles la estructuración del conocimiento, y con él, de la sociedad entera. En este mismo sentido se fundó la administración como disciplina científica (Forrester), ya desde sus orígenes hasta el día de hoy: Ford, Taylor, Fayol, hasta Porter, por ejemplo. En fin, exactamente con este espíritu se explicó a la naturaleza, ya sea con el sol como centro, o con la reina abeja u hormiga, o el rey león, alrededor de los cuales todo lo demás pivotaba. Todo, en resumen, fue la trasposición sin más de un modelo distintivamente antropológico y antropocéntrico al conjunto del universo, la naturaleza y el mundo.

Pues bien, específicamente las ciencias de la complejidad han venido recientemente a poner de manifiesto que, en verdad, la naturaleza, el mundo y el universo no tienen, en absoluto, una estructura jerárquica y piramidal. Precisamente por ello al mismo tiempo han emergido varias disciplinas y ciencias al interior de las ciencias de la complejidad, y varios enfoques y líneas de estudio al respecto. Primero, se trató del descubrimiento de sistema autoorganizados (Nicolis, Prigogine, 1994; Nicolis, 2007); seguidamente, el descubrimiento y explicación de la inteligencia de enjambre (Bonabeau et al., 1999), y el estudio de los cardúmenes, las escuelas de pájaros, las manadas; en el mismo sentido fue determinante el nacimiento de la ciencia de redes complejas (Watts, 2003; Strogatz, 2003; Barabasi, 2003), y la puesta en evidencia de redes libres de escala, redes de mundo pequeño y redes aleatorias. Sobre esta base, diversos otros descubrimientos, desarrollos e investigaciones emergieron conformando una atmósfera perfectamente novedosa cuyo resultado puntual consiste en la puesta en evidencia que mientras que los sistemas jerárquicos son exclusivos de la forma occidental de vida humana, hubo y hay otras formas de organización de la vida. Giramos a continuación la dirección en esta otra dirección.

\section{Las redes complejas y los temas de gestión}

Otras formas de organización del mundo y de las cosas son posibles. Si bien le corresponde el mérito a las ciencias de la complejidad haber puesto manifiestamente sobre la mesa, a plena luz del día, que los sistemas jerárquicos no constituyen, en absoluto, ni una regla ni una necesidad, originariamente, quien lleva la delantera en el descubrimiento de formas de organización no piramidales es la antropología. En efecto, ésta pone en evidencia que hubo y existen aún comunidades, culturas, pueblos y civilizaciones cuya forma de organización no es, en absoluto, jerárquica, sino heterárquica. Dejaré para la sección siguiente la discusión sobre la heterarquía. Por lo pronto quiero anticipar que la heterarquía es una forma de organización o de red compleja aun cuando la ciencia de redes complejas no haya formulado ni una sola palabra al respecto (Barrat et al., 2008). Me concentraré aquí, por lo pronto en las redes complejas con la idea de demostrar que es posible una gestión compleja que, consiguientemente, no sea vertical, piramidal, directiva. Por lo demás, la mejor introducción a las características y estructuras de la heterarquía es el estudio de las redes complejas.

El estudio de las formas de organización, primero, y luego, la consideración sobre si, cómo y por qué, algunas de estas formas pueden transformarse en otras es el objeto propio de la topología. Un campo que permanece ampliamente desconocido entre politólogos, administradores, economistas, sociólogos, principalmente.

No sin varios antecedentes, que conducen, retrospectivamente, hasta los trabajos pioneros de Euler, Riemann, Möbius, y Poincaré, entre otros, puede decirse que la topología nace propiamente gracias a S. Smale en 1966, cuando este gana la Medalla Fields. La forma primaria de topología es la llamada de topología de árbol, que expresa justamente a un sistema jerárquico (Maldonado, 2021). La topología puede ser vista como la transición de la geometría y el estudio de espacios y configuraciones a las transformaciones de las mismas. El lenguaje habitual es el de superficies, invariancia, espacios, estructuras, clases, mapas, conjuntos, diagramas, redes, y en un plano más desarrollado, hiperespacio, complejos algebraicos, homología, homeomorfismo (Alexandroff, 1961; Graham Flegg, 1974).

Pues bien, una estructura determinada puede, efectivamente, ser transformada en otra, incluso sin necesidad de que haya una ruptura en el espacio. Tal es exactamente la base del trabajo en topología; una idea fundamental que es ampliamente ignorada en campos de las ciencias sociales y humanas, específicamente en las arenas del institucionalismo, lato sensu. En primer lugar, eso: es perfectamente posible que una estructura determinada pueda ser transformada en otra; y en segunda instancia, se trata entonces de los juegos y posibilidades de justificación de la transformación. La topología no entra explícitamente en este terreno, aunque buena parte de la explicación estriba en el juego y los juegos de imaginación (Roberts, 2007). Pero el manejo del mundo, con sus miserias y sus grandezas sí demanda explícitamente una justificación. Particularmente cuando se trata de plantear la insostenibilidad, desde cualquier punto de vista, de una visión centralizada y piramidal de la realidad.

Otras topologías básicas son las topologías de estreIla, bus, anillo, malla, doble anillo, mixta, y totalmente conexa. Dicho de manera negativa: no es inevitable que la única, y ni siquiera la mejor, con cualquier justificación que se quiera aducir, forma de organización sea la topología árbol. Sólo este reconocimiento ya constituye un avance grande.

La gran contribución de las ciencias de la compleji- 
dad en general consiste en la puesta en evidencia de que la linealidad no es, desde ningún de vista, un fenómeno deseable ni necesario, en ningún sentido, ni en ningún plano o contexto que se considere. Por el contrario, la no-linealidad es la marca misma de la naturaleza, y aquella puede ser leída de diversas maneras al mismo tiempo: como atractor extraño, como fractalidad, como red compleja -específicamente, como red libre de escala-, como el proceso mismo mediante el cual in sistema determinado gana grados de libertad, y otras caracterizaciones. La complejidad de un fenómeno es directamente proporcional a los grados de libertad que tiene o que exhibe dicho fenómeno. Esta idea se origina en la física de sistemas autoorganizativos y alejados del equilibrio (= termodinámica del no-equilibrio) pero permea a numerosas ciencias y disciplinas y ha sido observada, recurrentemente, en la naturaleza, desde el comportamiento de moléculas y tejidos vivos, hasta las relaciones de diversas especies en un ecosistema determinado, hasta los sistemas de autoaprendizaje que se encuentran en la base de la robótica y la vida y la inteligencia artificiales.

El mundo descubierto por la ciencia de redes complejas es amplio, ubicuo y sorprendente a la vez. Quisiera destacar, de manera puntual, tres rasgos aquí. Estos son, la sincronización, la existencia de nexos libres de escala, y las conexiones que implican leyes de potencia. Estos tres rasgos son fundamentales para lograr una gestión modo complejo.

I) Sincronización. La sincronización comporta un tema perfectamente distinto al de la linealidad (o, si quiere, el de la linealización). Los sistemas simples están alineados; los sistemas complejos son sistemas sincronizados (Strogatz, 2003; Barabasi, 2011; cfr. Kuramoto model, web). La sincronización es un fenómeno que ha sido observado ya a partir de sistemas físicos, esto es, abióticos, pero que, a fortiori sucede en los sistemas orgánicos. Técnicamente, se trata del modelo Kuramoto, que estudia efectivamente transiciones irreversibles; estas tienen lugar tanto en el mundo físico como en el mundo de los fenómenos vivos. La sincronización es un fenómeno emergente espontáneo.

Evidentemente que la sincronización supone un comportamiento colectivo emergente, que no sabe para nada de centralidad, direccionamiento y demás, y que implica una pluralidad de agentes. Vale subrayar eso: la sincronización no implica, en absoluto, que haya un agente que sincronice (a otros); y que entonces derivativamente, organice, estructure. En otras palabras, la sincronización rompe en mil pedazos la idea de centralidad, verticalidad, lineamiento, planeación, liderazgo en cualquier acepción de la palabra, en fin, estrategia y demás herramien- tas propias de los estudios sobre gestión en sentido amplio.

Los fenómenos de sincronía han sido observados en toda clase de fenómenos, sistemas y comportamientos, desde la física a la química, desde la biología a los sistemas humanos. Simple y llanamente, si puede decírselo así, a la naturaleza en sentido amplio e incluyente, le encantan los procesos de sincronización. En el sistema solar o al interior del cuerpo humano, en la galaxia tanto como en metrónomos, en fin, en sistemas naturales o artificiales.

Como se desprende, sin dificultad, los fenómenos de sincronización comportan el reconocimiento de que la linealidad es un artificio, tanto como la necesidad de una cabeza, causa o agente que produzca la sincronía. Ciertamente que la sincronización es un descubrimiento reciente, pero el marco amplio en el que se sitúa es el del estudio de redes complejas.

ii) Redes libres de escala. En esta subsección queremos en realidad ocuparnos de los tres tipos principales de redes complejas, a saber: las redes libres de escala, las redes de mundo pequeño y las redes aleatorias. Las tres constituyen una sólida unidad consistente en mostrar, simple y llanamente que no todas las cosas están conectadas, sino, mucho mejor, que hay unas que están más conectadas con otras y otras más que están menos conectadas con las demás.

Propiamente hablando, toda red libre de escala es una red aleatoria cuya distribución corresponde a una ley de potencia. Por consiguiente, se trata del estudio de aquellas dinámicas y estructuras en las que existen algunos elementos diferenciales - propios de una ley de potencia- en el que todos cuentan, así sea con diferencias. De este modo deja de ser válido ese lenguaje según el cual hay algunas cosas o elementos o instancias "que son más importantes que otras". Puede decirse, sin la menor dificultad que muchas redes complejas son libres de escala.

Como se aprecia sin ambages, saber de redes complejas comporta la posibilidad -por decir lo menos-, de dejar de asumir que hay niveles o espacios más importantes que otros, sin que ello implique, en absoluto, que todos los espacios, niveles o instancias son igualmente importante, sin más. La marca distintiva de un sistema compleja es, sin la menor duda, la presencia de una ley de potencia. Sería deseable que en la consideración acerca de sistemas de organización de cualquier índole se trabajara y se supiera de leyes de potencia, un tema en cuya base se encuentra la ley de Zip y los trabajos sobre fractalidad (Mandelbrot, 1997). Aquello 
que emerge, consiguientemente, es la importancia de sistemas irregulares (Maldonado, 2020b). Una gestión modo complejo es gestión de irregularidades en el sentido técnico que se deriva de estas consideraciones.

iii) Leyes de potencia. Digámoslo de manera directa e inmediata. Las leyes de potencia dan lugar sistemas que técnicamente se denominan de criticalidad autoorganizada (SOC, por sus siglas en inglés), que es la forma misma como funciona la naturaleza (Bak, 1996). Todo parece indicar que las leyes de la naturaleza son simples, pero la naturaleza es compleja, lo que no es sino una manera de decir que la naturaleza en general se organiza autónoma, esto es, espontáneamente, en puntos y estados críticos a partir de los cuales tienen lugar transiciones de fase; esto es, transiciones de fase de primer orden y se dundo orden. En otras palabras, graduales y súbitas. El mundo es un entramado de sistemas de organización y no de caos, y todo tiene lugar de forma espontánea (order-for-free) (Kauffman, 2016).

Pensar en complejidad significa reconocer que existen fenómenos, estructuras y dinámicas espontáneas; esto es, que no obedecen, en manera alguna, al principio de causalidad. Esta no es sino una manera de decir que el azar cumple un papel constructivo en el comportamiento de los sistemas complejos, lo cual traducido al lenguaje de los sistemas humanos tiene un mensaje inmediato. Se trata del reconocimiento de que debemos poder confiar en fenómenos autoorganizativos, tanto más si ellos son la regla en la naturaleza. Los sistemas jerárquicos son la evidencia de un sistema de desconfianza, recelo, sospecha y recusaciones, todo un ambiente manifiestamente enfermizo y, ulteriormente, punitivo y policivo.

De esta suerte, las leyes de potencia, que se encuentran en la base de los sistemas autoorganizados -técnicamente dicho: sistemas de criticalidad autoorganizada-, constituyen la marca de un ambiente de confianza en los procesos y dinámicas (Luhmann, 1996), y por tanto, ponen de manifiesto la futilidad, por así decirlo, de sistemas de control. Simple y llanamente, en la naturaleza no existen, en modo alguno, sistemas control; antes bien, se trata de sistemas de regulación, de retroalimentación -positiva y negativa-, de co-evolución, en fin de aprendizaje recíproco y de adaptación. Precisamente por ello la ciencia de punta en general deja de hablar de leyes a cambio de patrones. Las leyes controlan -véase su origen semántico en el sistema legal y punitivo cuyos orígenes remiten a los fundamentos mismos de Occidente-, en tanto que los patrones (patterns), son evolutivos. En la base de la fractalidad se halla un sistema de iteración. No otra cosa es la ecuación que produce fractales de Mandelbrot, a partir de las ecuaciones de Julia. La iteración es una operación que se hace sobre el espacio, análoga a las operaciones clásicas de reflexión, traslación y demás. La iteración de una ecuación produce patrones sugestivos: los fractales (pero lo mismo puede decirse a numerosos conjuntos (Cantor, Peano, Serpiensky, y otros).

Como se aprecia, las redes complejas, los sistemas de sincronía y las leyes de potencia constituyen una férrea unidad cuyo resultado es la emergencia de fenómenos autoorganizativos, esto es, no centralizados ni piramidales, en los que el control deja de existir o de jugar cualquier papel. En otras palabras, el estudio de la complejidad consiste, simple y llanamente, en observar de manera desprevenida pero alerta, a los fenómenos, dinámicas y estructuras de la naturaleza. De allí parecen emerger una serie de mensajes altamente significativos. Pues bien, lo mejor de la investigación de punta (spearhead research) pasa, nuclearmente, por el estudio y comprensión de los sistemas de complejidad creciente. Importantes como son, todos los temas de gestión -esto es, de gobierno, administración, gobernanza, y otros semejantes- deben saber de complejidad si, por decir lo menos, quieren generar un ambiente de confianza ("cultura organizacional" con base en la confianza), antes que en I sanción, la sospecha y el castigo. Las consecuencias que se siguen de un sistema de autoorganización son refrescantes y relajantes al mismo tiempo; y por ello mismo, se traducen en calidad de vida y en dignidad. Esto no es poco, en medio de tiempos de tensión, de inestabilidad, de mucha zozobra y desasosiego todo lo cual se condensa en el concepto de competencia y competitividad.

Dicho de manera escueta, una gestión compleja tiene lugar mucho más a la manera de la naturaleza que de la cultura, sin que ello implique una afirmación dualista. En una palabra, como se sigue de las líneas anteriores, una gestión compleja es un sistema carente de control. Una idea ciertamente escandalosa o contraintuiva cuando se la mira con los ojos del pasado o de la ciencia normal o del sentido común. Volveremos sobre esta idea.

\section{Heterarquía: un asunto complejo, en el sentido preciso de la palabra}

El desplazamiento, radical y, queremos pensarlo, irreversible, de sistemas de control centralizado hacia sistemas carentes de control puede ser igualmente entendido gracias al estudio de la heterarquía. Este desplazamiento es posible, y queremos incluso sugerir que es deseable dada toda la historia de violencia, abierta y simbólica de la historia de Occidente con sus conse- 
cuencias de inequidad, injusticia, pobreza y violación de los derechos humanos (= dignidad y calidad de la vida).

Mientras que la jerarquía es un concepto que permea a la totalidad de la civilización occidental -abierta o tácitamente-, la antropología y la historia -esas dos ciencias políticamente incorrectas-, han venido a poner de manifiesto, recientemente, el papel de la heterarquía en la forma de organización y de gobierno de pueblos, culturas y civilizaciones, digamos, no occidentales y de manera profusa (Kurnik and Baron, 2016; Langebek, 2019; Hüsken, 2019; Izquierdo y de la Cueva, 2018; Ugalde y Landázuri Narváez, 2016). El mensaje que inmediatamente salta ante la mirada atenta es el de la importancia de la antropología, la historia e incluso la microhistoria. Digámoslo en términos fuertes: mucho antes y por encima de los estudios ingenieriles, administrativos, financieros, de política pública y de estrategia, en cualquier acepción de la palabra.

Las comunidades, sociedades, pueblos y culturas que se han organizado en términos de heterarquía reconocen que la jerarquía es una función, transitoria, por consiguiente, no un estado, y que por su impermanencia está definida a partir de las circunstancias, los eventos, las contingencias o las necesidades, según el caso. De manera significativa, los sistemas heterárquicos han sido observados también en numerosas especies de animales (Sheldrake, 2012). De base, las heterarquías han sido reconocidas ya desde la teoría ecológica, por lo menos en los últimos veinte años (Cumming, 2016).

Pues bien, quiero argumentar que la heterarquía sí contradice a la jerarquía (en contraste con la tesis doctoral de Bernal, cfr. file://C:/Users/cemca/Downloads/Bernal\%20Velez.pdf), de la misma manera como las redes complejas contradicen también a la jerarquía. Una gestión modo complejo es gestión a la manera de la naturaleza, en marcado contraste con la imagen distintivamente antropocéntrica en la que el mundo y la sociedad se organizaron en el pasado, hasta el día de hoy, en Occidente. Dicho sin más, una gestión compleja es autogestionaria, en el modo mismo como la naturaleza es un sistema autoorganizado.

El estudio de las heterarquías es el resultado de trabajos en antropología, etnología, etnografía, epigrafía y paleografía, principalmente, específicamente a partir de comienzos del siglo XXI, si bien existen algunos trabajos, incipientes, de finales del siglo XX. Si la administración, en el sentido amplio de la palabra es la hija (putativa) de la ingeniería clásica, de la física clásica y de la mecánica estadística -eso son Forrester, Ford, Taylor, Fayol y toda la historia que conduce incluso, hoy en día, hasta Porter-, a fin de aprender acerca de redes y complejas bien valdría la pena que supiera de antropología, sociología, microhistoria, mucha psicología y otros campos próximos y semejantes. No en última instancia, la administración en el sentido más amplio e incluyente de la palabra debería poder saber también de ecología. En verdad, en la naturaleza no existen jerarquías, y ciertamente no verticalidad y centros ( $y$, relativamente, periferias). Por el contrario, la naturaleza se organiza en términos de flujos, correlaciones, bucles de retroalimentación positiva y negativa, transiciones de fase, ciclos y dinámicas no-lineales, coevolución, coaprendizaje, mutualismo, comensalismo y cooperación. Y por ello mismo la naturaleza funciona muy bien; de lejos, inmensamente mejor que la cultura humana; por lo menos, sin duda alguna, la de Occidente, que incluye al sistema de libre mercado y al neoliberalismo como sus mejores y más acabadas expresiones. La naturaleza sabe organizarse de forma bastante más armónica que como lo han hecho los seres humanos, por lo menos en los últimos cinco mil años. La heterarquía es organización de la cosa humana en términos de sistemas naturales.

En verdad, en los sistemas heterárquicos existe, naturalmente, rendición de cuentas (logos didomai; accountability), pero hacia abajo y hacia los lados. No existen instancias superiores en las heterarquías, sólo horizontalidad y responsabilidad hacia las instancias así llamadas inferiores. Al fin y al cabo, lo que hay a los lados y hacia abajo es gente, seres humanos, procesos vivos, y es hacia ellos que se distribuyen las responsabilidades y la rendicón de cuentas del buen manejo de los asuntos que tienen que ver con el convivio, en sentido amplio, profundo y fuerte.

De lejos, el tema más amplio en las heterarquías, tanto como en las redes sociales es la confianza. En contraste, los sistemas jerárquicos están definidos por el control y la manipulación. En efecto, temas como liderazgo (de distinto tipo), planeación, estrategia, Misión, Visión, himno, objetivos, planes y programas, bandera y otros más se definen frontalmente de cara al control: son mecanismos de control. Pues bien, no hay nada más alejado de la complejidad y de la vida.

Las redes complejas -una de las ciencias de la complejidad- y las heterarquías son sistemas autogestionarios; en el horizonte, y esto es algo que cuesta a muchos, entender, son igualmente sistemas anárquicos; esto es, sistemas libres, sin controles, no verticales, no directivos, punitivos y policiales. En el lenguaje de la complejidad un sistema autogestionario es un sistema autoorganizativo. La organización se define en función de las necesidades, las circunstancias, los avatares. No existen funciones fijas, y tampoco puestos determinados de actividad. Un sistema complejo es esencialmente adaptativo porque tiene la capacidad de aprender (Maldonado, 2021).

Existen, alrededor del mundo, numerosas experiencias autogestionarias: en Francia tanto como en la India, en Japón y en Nueva York, y desde luego, en México, Brasil o Colombia, por ejemplo. (Véase: algunos 
sistemas autogestionarios, al final, en las referencias bibliográficas. Un texto que compile y estudie entonces las diversas experiencias autogestionarias alrededor del mundo sería el objeto de un artículo aparte). En semejantes experiencias de autogestión emergen sistemas alternativos de educación, de economía, de formas de vida, de relacionamientos, axiológicas y éticas de sistemas monetarios, en fin, de relaciones con la naturaleza. Lo verdaderamente interesante radica en el hecho de que todas las experiencias autogestionarias que están teniendo lugar en el mundo se observan unas a otras, aprenden, se multiplican se fortalecen y comienzan a convertirse en evidencias de un nuevo tipo de civilización en emergencia ${ }^{1}$.

En cualquier caso, una gestión modo complejo tiene lugar a través de cualesquiera de los planos mencionados: como una red compleja o bien como heterarquía. Pues bien, hay un elemento absolutamente importante que define a las redes complejas y a las heterarquías, en marcado contraste con los sistemas jerárquicos (Cumming, 2016). Se trata de la importancia del open data. En efecto, la información abierta, compartida, horizontal o incluso con un compromiso de apertura hacia los niveles inferiores es algo que no existe, en absoluto en los sistemas piramidales y directivos. Todo lo contrario. Las asimetrías de información, como ya está suficientemente estudiado (Baker, 2008; Snowden, 2019) implican sistemas de control, de vigilancia, de manipulación y de violencia.

Una política de datos abiertos -Open Access- comporta un sistema de confianza, de transparencia, y una forma de democracia como jamás existió en la historia de Occidente. Y que, sin embargo, sí existió entre las culturas mesoamericanas: los olmecas y toltecas, los mayas, los muiscas y los incas, notablemente. Las redes complejas y la heterarquía significan, simple y sencillamente, un respeto hacia los demás, en el que la ética y la política no son simplemente discurso y representaciones, sino compromiso y actividad co-responsable. Lo apasionante es que experiencias semejantes de heterarquía comienzan a hacerse extensivas, hoy en día, a otras culturas y en otras geografías (Wynnycky, 2020; Fichera, 2020).

La implementación de un sistema de gestión modo complejo es el verdadero reconocimiento del otro en sus diferencias y comunidad, y la conformación de una atmósfera de transparencia. Como se aprecia, una historia radicalmente posible con respecto a la historia de los últimos dos mil quinientos años. Un motivo de optimismo.

En verdad, aquello de lo cual se trata verdadera-

1 Trabajo actualmente en un texto que se ocupa exactamente de este tema para un capítulo de libro al que he sido invitado y que se publicará en la Universidad Nacional de Colombia en el año 2022, en torno a bioeconomia. mente en las tomas de decisión, en las acciones y en las formas de organización es de vida; mucho más que de recursos, dineros, energías, materiales, bienes, cadenas de valor, clima y cultura organizacional y demás. Una democracia verdadera, al interior de las empresas, las organizaciones, las instancias públicas y de gobierno, al interior de las iglesias, los ejércitos y las fuerzas de policía, los equipos deportivos y demás, no es otra cosa que un ejercicio de datos abiertos y acceso abierto a la información, y las acciones que se siguen de ello. Sin jerarquías, sin secretismo, sin ocultamientos de ninguna índole. Como se aprecia sin dificultad existe una doble o recíproca puerta de acceso a una gestión modo complejo; o bien desde políticas abiertas y transparentes de información en toda la línea de la palabra, o bien en términos de organización de estructuras, niveles, modos, espacios, dinámicas y responsabilidades en términos de redes libres de escala tanto como de heterarquía.

Sin dilaciones, un modo de gestión semejante es el modo mismo de funcionamiento y estructuración de la naturaleza; esto es, de los ecosistemas, los nichos ecológicos, los biomas, en fin, los microclimas. Contra políticas de corte ingenieril del tipo WeWork y TheBestPlacetoWork, y otros semejantes, un buen "clima organizacional" es, sin la menor duda, un clima de transparencia, no de opacidad, de acercamiento y no de distancias, sino de horizontalidad; y siempre no de jerarquías. Una cultura organizacional no puede ser, en absoluto, distinta a una cultura de vida, lato sensu, y en el sentido más fuerte de la palabra; esto es, una cultura de gratificación, exaltación, posibilitamiento de la vida misma, libertad, dignidad, calidad y alegría de vida. Como se aprecia, todo lo opuesto a las ingenierías en boga. Una ingeniería modo complejo -que se dice entonces como ingeniería de sistemas complejos (complex engineering systems) (Maldonado, Gómez Cruz, 2012; Maldonado-Gómez-Cruz, 2011)- invita a dirigir la mirada, mucho más que hacia un cambio organizacional simplemente, hacia una transformación civilizatoria.

En una palabra, el tema de base de una gestión modo complejo consiste en el reconocimiento de que de lo que se trata es de transformar las organizaciones como una forma de transformar el mundo. De abajo hacia arriba, de un lado hacia el otro, y sin que exista, física y sobre todo simbólicamente, un arriba. En el universo, hay que recordarlo, no existe arriba ni abajo, a la izquierda o a la derecha. El universo no se encuentra en ninguna parte. Él mismo es el tiempo y el espacio. El universo, la naturaleza: lo mismo da.

Sin editorializar, otra democracia es posible, otras formas de organización son posibles, otros estilos, estándares y formas de vida son posibles. Todo ello pasa por las formas de estructura y dinámica de las organizaciones humanas; desde la familia hasta los asuntos 
mundiales, pasando por los niveles local, regional y nacional. La historia de la violencia -abierta o tácita, física o simbólica- de los sistemas jerárquicos y piramidales debe ser dejada atrás irreversiblemente. Se trata, sin más ni más, de mirar hacia adelante una historia de gratificación de la vida - de la vida humana tanto como de la vida en general sobre el planeta-.

\section{Conclusiones: una gestión compleja es gestión de vida}

El control jerárquico corresponde a una organización del mundo con base en la geometría euclidiana. Pues bien, hay que decir que la geometría de la naturaleza (Mandelbrot, 1997), tanto como la geometría de los pueblos primitivos o aborígenes son una geometría no-euclidiana. Una organización del mundo y la sociedad en términos piramidales es sencillamente antinatural, y por ello mismo, violenta. He argumentado aquí en favor de una organización del mundo acorde a la naturaleza. Las dos expresiones que inmediatamente aparecen son las redes complejas y la heterarquía. Occidente no supo, en su historia oficial, nunca acerca de las mismas. Dicho esto, la heterarquía, las redes complejas y la autoorganización constituyen alternativas a la historia occidental de organizar las cosas - las humanas y las naturales.

Afirmar la necesidad de estructuras jerárquicas es un total error; el problema estriba en realidad en que un error semejante se traduce inmediatamente en la implementación de un sistema de cosas injustas y que genera sufrimiento en las gentes. Un sistema cualquiera que produzca sufrimiento entre los seres humanos, y de consuno, destrucción de la naturaleza carece de cualquier justificación. Se impone, sin restricciones, la transformación de un sistema semejante. Pues bien, es exactamente en este punto en donde entra la complejidad.

Una gestión modo complejo -gestión de la educación, de los asuntos espirituales, de la economía, de los procesos psicológicos y emocionales, incluso de los temas militares o de policía cuando estos son necesarios, una organización de los asuntos públicos y de los asuntos comunes (commons) (Ostrom, 1995) ${ }^{2}$; en fin, una gestión y gobierno de los asuntos humanos y no humanos- radica en el reconocimiento explícito acerca de la vacuidad de un sistema organizacional de tipo vertical y directivo de gestión, y el Ilamado, por el contrario, a la autoorganización, la autogestión y la confianza, todo lo cual no es, simpe y Ilanamente sino un llamado a la autonomía y el aprendizaje.

2 Hay que decir que E. Ostrom no sabe para nada de heterarquía ni de redes complejas. La referencia aquí a su trabajo se basa en la importancia de los bienes comunes, en contraste con los bienes públicos y los bienes privados.
La complejidad comporta un modo perfectamente distinto de pensar y de vivir. Dicho en términos epistemológicos, se trata de aprender a pensar sin categorías, pues las categorías clasifican ordenan, jerarquizan, y así, congelan las cosas. Pensar sin categorías se traduce, existencial o experiencialmente como una relación horizontal, sin instancias superiores en manera alguna. Pues bien, la historia de la ciencia y la filosofía coinciden tanto en la importancia y la necesidad de las categorías, como en formas de vida que asumen, abierta o tácitamente, centralidades y jerarquías. En contraste, se trata de comenzar a aprender a pensar y vivir como la naturaleza, lo cual es bastante más y muy distinto simplemente a la ciencia, la tecnología y la filosofía. Un destello de sabiduría emerge entonces. Es, justamente, lo que comporta una gestión modo complejo.

\section{Bibliografía}

Aime, F., Humphrey, S., Scott Derue, D., and Paul, J. B., (2014). "The riddle of heterarchy: power transitions in cross-functional teams", en: The Academy of Management Journal, April, Vol. 57, No. 2, pp. 327-352; doi: http://dx.doi.org/10.5465/amj.2011.075

Alexandroff, P., (1961). Elementary Concepts of Topology. New York: Dover

Annushkina, O. E., Regazz, A., (2020). The Art of Going Global A Practical Guide to a Firm's International Growth. Palgrave Macmillan; doi: https://doi. org/10.1007/978-3-030-21044-1

Baker, S., (2008). The Numerati. London: Jonathan Cape Barabasi, A.-L., (2003). Linked. How Everything is Connected to Everything Else and What It Means for Business, Science, and Everyday Life. New York: Plume

Barabasi, A.L., (2011). Bursts. The Hidden Patterns Behind Everything We Do, from Your E-mail to Bloody Crusades. New York: Plume

Barrat, A., Barthélemy, M., Vespignani, A., (2008). Dynamical Processes on complex networks. Cambridge: Cambridge University Press

Bonabeau, E., Dorigo, M., Theraulaz, G., (1999). Swarm Intelligence. From Natural to Artificial Systems. New York-Oxford: Oxford University Press

Byers, A. M., (2011). Sacred Games, Death, and Renewal in the Ancient Eastern Woodlands: The Ohio Hopewell System of Cult Sodality Heterarchies. AltaMira Press

Cayón, L., (2020). "Disputas fraternas e chefia bicéfala: Hierarquia e heterarquia no Alto Rio Negro", en: Rev. antropol. (São Paulo, Online), v. 63 n. 2: e151160; doi: http://dx.doi.org/10.11606/ 2179-0892. ra.2020.171366

Chandrashekar Smitha, K., (ed.), (2017). Entrepreneurial Urbanism in India The Politics of Spatial Restructuring and Local Contestation. Springer Verlag; doi: 10.1007/978-981-10-2236-4 
Crumley, C. L., (2005). "Remember How to Organize: Heterarchy Across Disciplines", en: Nonlinear Models for Archaeology and Anthropology: Continuing the Revolution, edited by Christopher S. Beekman. Taylor \& Francis Group

Cumming, G. S., (2016). "Heterarchies: Reconciling Networks and Hierarchies. Review", en: Trends in Ecology \& Evolution, Agosto, Vol. 31, No. 8, págs. 622-632; doi: http://dx.doi.org/10.1016/j.tree.2016.04.009

Fichera, M., (2020). "Solidarity, Heterarchy, and Political Morality", en: Jus Cogens (2020) 2:301-311; doi: https://doi.org/10.1007/s42439-020-00019-w

Foccroulle, B., Legros, R., Todorov, T., (2005). El nacimiento del individuo en el arte. Buenos Aires: Ed. Nueva Visión

Gauthier, D., (1998). Egoísmo, moralidad y sociedad liberal. Barcelona: Paidós

Goody, J., (2006). The Theft of History. Cambridge: Cambridge University Press

Grahamm Flegg, H., (1974). From Geometry to Topology. New York:; Dover

Hüsken, Th., (2019). Tribal Politics in the Borderland of Egypt and Libya. Palgrave Macmillan

Izquierdo y de la Cueva, A. L., (2018). "Heterarquía y unidades corporativas. Instituciones del gobierno interno maya", en: Estudios de cultura maya LI, págs. 11-42; doi: 10.19130/iifl.ecm.2018.51.855

Kauffman, S., (2016). Humanity in a Creative Universe. Oxford: Oxford University Press

Kurnick, S., and Baron, J., (2016). Political Strategies in Pre-Columbian Meso-America, Ed. S., Boulder, University Press of Colorado

Langebaek, C., (2019). Los Muiscas. La historia milenaria de un pueblo chibcha. Bogotá: Debate

Lasa López, A., (2018). "Potencialidades del pilar europeo de derechos sociales en la praxis jurisprudencial del juez de Estrasburgo: entre la heterarquía vínculo social-vínculo económico y la disfuncionalidad", en: Teoría y Realidad Constitucional, núm. 42, 2018, pp. 609-631

Lévèque, P., (Dir.)., (2012). Las primeras civilizaciones. De los despotismos orientales a la ciudad griega. Madrid: Akal

Luhmann, N., (1996). Confianza. Barcelona: Anthropos

Macpherson, C. B., (2005). La teoría política del individualismo posesivo. Madrid: Trotta

Maldonado, C. E., (2021). "Las organizaciones inteligentes son organizaciones que saben de complejidad", en: Revista Ciencias de la Complejidad, No. págs. 81-92, doi: https://10.48168/cc012021-008

Maldonado, C. E., (2020a). Occidente, la civilización que nació enferma. Bogotá: Desde Abajo

Maldonado, C. E., (2020b) "Ciencias sociales irregulares", en: Cinta de moebio 68: 146-155; doi: https:// doi.org/10.4067/S0717-554X2020000200146; disponible en: file:///C:/Users/cemca/Downloa ds/58586-193-199852-1-10-20200914.pdf
Maldonado, C. E., Gómez-Cruz, N.-, (2012). (2012f) "The Complexification of Engineering", en: Complexity, Vol. 17, Issue 4 (March-April), pp. 8-15; http://onlinelibrary. wiley.com; doi: http/10.1002/cplx.v17.4/issuetoc

Maldonado, C. E., Gómez-Cruz, N., (2011). "Biological Computation: A Road to Complex Engineered Systems"; capítulo de libro, en: Sayama, H., Minai, A., A., Braha, D., and Bar-Yam, Y., (Eds.), Unifying Themes in Complex Systems. Volume VIII: Proceedings of the Eighth International Conference on Complex Systems. New England Complex Systems Institute Series on Complexity, págs. 918-927, NECSI Knowledge Press, ISBN 978-0-9656328-4-3 http://necsi.edu/events/ iccs2011/proceedings.html

Mandelbrot, B., (1997). La geometría fractal de la naturaleza. Barcelona: Tusquets

Mezza-García, N., Maldonado, C. E., (2015). "Crítica al control jerárquico de los regímenes políticos: complejidad y topología", en: Desafíos, (27-1), págs. 121-158, ISSN 0124-4035; ISSNE 2145-5112; doi: https://doi. org/10.12804/desafios27.01.2015.04

Nicolis, G., Nicolis, C., (2007). Foundations of Complex Systems. Nonlinear Dynamics. Statistical Physics, Information and Prediction. World Scientific

Nicolis, G., Prigogine, I., (1994). La estructura de lo complejo. En el camino hacia una nueva comprensión de las ciencias. Madrid: Alianza

Ostrom, E., (1995). Governing the Commons. The Evolution of Institutions for Cllective Action. Cambeidge: Cambridge University Press

Pini-Fitzsimmons, J., Knott, N. A., and Brown, C., (2021). "Heterarchy Reveals Social Organization of a Smooth Stingray (Bathytoshia brevicaudata) Population in a Provisioned Food Context", en: Frontiers in Marine Science, abril, vol 8, article 641761; doi: https://doi. org/10.3389/fmars.2021.641761

Roberts, S., (2007). King of Infinite Space. Donald Coxeter, The Man Who Saved Geometry. London: Profile Books

Schoelhammer, S., (2020). Innovation Exposed. Case Studies of Strategy, Organization and Culture in Heterarchies. Springer Verlag; doi: https://doi. org/10.1007/978-3-658-29335-2

Sheldrake, R., (2012). The presence of the past. Morphic resonance and memory of nature. Rochester-Toronto: Park Street Press

Snowden, E., (2019). Vigilancia permanente. Bogotá: Planeta

Strogatz, S., (2003). Sync. How Order Emerges from Chaos in the Universe, Nature, and Daily Life. New York: Theia

Ugalde, M. F., y Landázuri Narváez, C., (2016). "Sociedades heterárquicas en el Ecuador preincaico: estudio diacrónico de la organización política caranqui", en: Revista española de antropología americana, vol. 46, págs. 197-218; doi: http://dx.doi.org/10.5209/ 
REAA. 58294

Watts, D. J., (2003). Six Degrees. The Science of a Connected Age. New York-London: W. W. Norton \& Company

Wynnycky, M., (2020). "Unravelling the Ukrainian Revolution: "Dignity," "Fairness," "Heterarchy," and the Challenge to Modernity", en: Kyiv-Mohyla Humanities Journal 7, págs. $123-140$

Páginas web:

Kuramoto model: A non-linear dynamics excercise: ht- tps://www. youtube.com/watch?v=430IzJ80yGM Algunos sistemas autogestionarios:

https://www.restosducoeur.org/

https://www. awesomefoundation.org/en/projects/66523-barter-market

https://asia.nikkei.com/Economy/The-truth-behind-India-s-new-barter-economy

https://en.wikipedia.org/wiki/List_of_alternative_universities

https://zad.nadir.org/?lang=es 